Pacific Journal of Mathematics

ON THE LEAST NUMBER OF FIXED POINTS FOR INFINITE 


\title{
ON THE LEAST NUMBER OF FIXED POINTS FOR INFINITE COMPLEXES
}

\author{
SHI GEN-HUA
}

Let $K$ be a connected infinite and locally finite simplicial complex. The main theorem of this paper is the following: let $L$ be a two-dimensionally connected infinite subcomplex of $K$, whose boundary $\dot{L}$ in $K$ consists of vertices only, and $f:|K| \rightarrow|K|$ be a map. Then there exists a map $F:|K| \rightarrow|K|$, that has the following properties: (1) $F \cong f$ rel $\overline{|K-L|}$; and, (2) $F$ has no fixed point on $|L|-|\dot{L}|$.

The main theorem implies that if an infinite and locally finite complex $K$ is two dimensionally connected, then the least number of fixed points of any mapping class from $|K|$ to itself is null. At the same time, the main theorem also enables us to compute the least number $m(K)$ of the fixed points of the identity mapping class of $|K|$ by means of the following result: $m(K)$ is equal to the least number $n(K)$ of the fixed points of the good displacements of the welding set $\dot{M}(K)$ of $K$, where $\dot{M}(K)$ is the set of the boundary vertices of all these maximal two-dimensionally connected and finite subcomplexes of $K$.

In this paper, an infinite complex means a complex whose simplices are countable infinite. On the other hand, a locally finite complex means a complex $K$ satisfying the following two conditions: For each simplex $\sigma$ of $K, \mathrm{St}_{K}(\sigma)$ consists of number of finite simplices and $\left|\mathrm{St}_{K}(\sigma)\right|$ is an open subset of $|K|$. The second condition means the topology of $|K|$ is the weak topology. If $x$ is a point of $|K|$, then it belongs to just one simplex of $K$ which is called the carrier of $x$ and is denoted by $\operatorname{Tr}_{K}(x)$. A complex $K$ is called two-dimensionally connected if for any two maximal simplices $\sigma$ and $\tau$ of $K$, there are simplices of $K$

$$
\sigma=\sigma_{0}, \sigma_{1}, \cdots, \sigma_{n-1}, \sigma_{n}=\tau
$$

such that $\sigma_{i-1}$ and $\sigma_{i}, i=1, \cdots, n$, have a common face of dimension greater than zero.

Suppose that $M$ is a subset of $|K|$ and that $f: M \rightarrow|K|$ is a map such that $\overline{\operatorname{Tr}_{K}(x)} \cap \overline{\operatorname{Tr}_{K}[f(x)]} \neq \phi$ for any $x \in M$, then we say that $f$ satisfies $S(K)$ on $M$. The following Lemma 1 is the generalization of Lemma 2.3 and Lemma 1.3 of [6].

LEMMA 1. Let $K$ be a locally finite complex and $\tau$ the common face of its maximal simplices $\sigma_{1}$ and $\sigma_{2}$, where the dimension of $\tau$ 
is greater than zero. Suppose we are given points $A \in \sigma_{1}, B \in \tau$ and a map $f:|K| \rightarrow|K|$ such that $A$ is an isolated fixed point of $f$ and it is the only fixed point of $f$ on $[A, B]$. Then we can find a map $F:|K| \rightarrow|K|$ and $\delta>0$ such that:

$$
F \cong f \operatorname{rel}[|K|-U([A, B], \delta)]
$$

and $F$ on $U([A, B], \delta)$ has only one fixed point $C$ belonging to $\sigma_{2}$. If $f$ satisfies $S(K)$ on $[A, B]$ then $F$ satisfies $S(K)$ on $\bar{U}([A, B], \delta)$.

Lemma 2. Let $K$ be a locally finite complex and $f:|K| \rightarrow|K|$ be a map. Then there is a map $F, F \cong f:|K| \rightarrow|K|$ such that each fixed point of $F$ is isolated and lies in a maximal simplex of $K$.

Proof. We can find a simplicial approximation $q: R \rightarrow K$ to $f$, where $R$ is a barycentric subdivision of a subdivision $H$ of the complex $K$. We first prove that $q$ has a maximum of one fixed point on the closure of each simplex of $R$ as follows. If $\sigma^{n}$ is a simplex of $R$ and $x_{1}, x_{2}$ are two fixed points of $q$ such that the open segment $\left(x_{1}, x_{2}\right) \subset \sigma^{n}$ belongs to $\sigma^{n}$, then the straight line $y=t x_{1}+$ $(1-t) x_{2}$ intersects $\dot{\sigma}^{n}$ at two points $y_{1}$ and $y_{2}$, which are fixed points of $q$. Because $x_{i}$ is a fixed point of the simplicial map $q$, then $\left|\operatorname{Tr}_{R}\left(x_{i}\right)\right| \subset\left|\operatorname{Tr}_{H}\left(x_{i}\right)\right|$, so the dimension of $\operatorname{Tr}_{H}\left(x_{i}\right)$ is $n$. The dimension of the carrier of $\left(x_{1}, x_{2}\right)$ in $H$ is $n$. Similarly, we have $\left|\operatorname{Tr}_{R}\left(y_{i}\right)\right| \subset$ $\left|\operatorname{Tr}_{H}\left(y_{i}\right)\right|$, so the dimension of $\operatorname{Tr}_{H}\left(y_{i}\right)$ is equal to the dimension of $\operatorname{Tr}_{R}\left(y_{i}\right)$ and less than $n$, for $i=1,2$. Since $R$ is the barycentric subdivision of $H, \sigma^{n}$ has a face $\sigma^{n-1}$, such that all the points of $\bar{\sigma}^{n}$ which have the carrier in $H$ of dimension less than $n$ belong to $\bar{\sigma}^{n-1}$. This fact implies that $y_{1}, y_{2} \in \bar{\sigma}^{n-1}$, which is a contradiction, because then we would have $\left(x_{1}, x_{2}\right) \subset \bar{\sigma}^{n-1}$.

Next we denote all the fixed points of $q$ as $x_{1}, x_{2}, \cdots$, so:

$$
\left|\operatorname{St}_{R}\left[\operatorname{Tr}_{R}\left(x_{i}\right)\right]\right| \cap\left|\operatorname{St}_{R}\left[\operatorname{Tr}_{R}\left(x_{j}\right)\right]\right|=\phi \text {, for } i \neq j \text {. }
$$

We choose $\delta_{i}>0, i=1,2, \cdots$, such that:

$$
\bar{U}\left(x_{i}, \delta_{i}\right) \subset\left|\operatorname{St}_{R}\left[\operatorname{Tr}_{R}\left(x_{i}\right)\right]\right|, i=1,2, \cdots,
$$

then:

$$
\bar{U}\left(x_{i}, \delta_{i}\right) \cap \bar{U}\left(x_{j}, \delta_{j}\right)=\phi, i \neq j .
$$

From [1] (Kapitel 14) we can find the maps $g_{i}: \bar{U}\left(x_{i}, \delta_{i}\right) \rightarrow|K|$ with $\varepsilon_{i}=\sup \left\{\rho\left[q(x), g_{i}(x)\right] \mid x \in \bar{U}\left(x_{i}, \delta_{i}\right)\right\}$, where $\rho$ is the metric of $|K|$, with $\varepsilon_{i}$ sufficiently small so that the following three conditions are satisfied:

(1) $\left.\overline{\operatorname{Tr}_{K}\left[g_{i}(x)\right]} \cap \overline{\operatorname{Tr}_{K}[q(x)}\right] \neq \phi$, for all $x \in \bar{U}\left(x_{i}, \delta_{i}\right)$; 
(2) each fixed point of $g_{i}$ is isolated and lies in a maximal simplex of $R$ as well as in $\bar{U}\left(x_{i}, \delta_{i} / 2\right)$; and,

(3) $\alpha\left[q(x), g_{i}(x),\left(2-2 \rho\left(x, x_{i}\right) / \delta_{i}\right) t\right] \neq x$, for all $0 \leqq t \leqq 1$ when $\delta_{i} / 2 \leqq \rho\left(x, x_{i}\right) \leqq \delta_{i}$.

Using the short homotopy $\alpha$ of Lemma 1.1 of [6] we define

$$
f_{t}(x)=\left[\begin{array}{l}
q(x), x \in|K|-\bigcup_{i} U\left(x_{i}, \delta_{i}\right), \\
\alpha\left[q(x), g_{i}(x),\left(2-2 \rho\left(x, x_{i}\right) / \delta_{i}\right) t\right], \delta_{i} / 2 \leqq \rho\left(x, x_{i}\right) \leqq \delta_{i}, \\
\alpha\left[q(x), g_{i}(x), t\right], 0 \leqq \rho\left(x, x_{i}\right) \leqq \delta_{i} / 2,
\end{array}\right.
$$

so $f_{t}$ is a homotopy between $q$ and $f_{1}$. Finally, let $F=f_{1}$, then each fixed point of $F$ is isolated and lies in a maximal simplex of $K$.

Lemma 3. Assume that $K$ is a locally finite complex, $M$ is a subcomplex consisting of vertices only, and that $g=M \rightarrow|K|$ is a map satisfying $S(K)$ on $M$. Then there is a map $F_{1}:|K| \rightarrow|K|$ that has the following properties:

(1) $F_{1}$ satisfies $S(K)$ on $|K|$;

(2) $F_{1}(x)=g(x)$, for all $x \in M$; and,

(3) each fixed point of $F_{1}$ on $|K|-M$ is isolated and lies in a maximal simplex of $K$.

Proof. In the proof of Lemma 2, let $f=1$; thus we can choose $\varepsilon_{i}$ to be sufficiently small to ensure that $F(x)$ satisfies $S(K)$ on $|K|$. Since $M$ consists of vertices of $K$, then

$$
\overline{\operatorname{Tr}_{K}(x)} \cap \overline{\operatorname{Tr}_{K}[g(x)]} \cap \overline{\operatorname{Tr}_{K}[F(x)]} \neq \phi,
$$

for all $x \in M$. Writing $M=\left\{y_{1}, y_{2}, \cdots\right\}$, we can find $\eta_{i}>0$, that have the following properties:

$$
\begin{aligned}
& \bar{U}\left(y_{i}, \eta_{i}\right) \cap \bar{U}\left(y_{j}, \eta_{j}\right)=\phi, i \neq j ; \\
& \bar{U}\left(y_{i}, \eta_{i}\right) \subset \operatorname{St}_{K}\left(y_{i}\right), F\left[\bar{U}\left(y_{i}, \eta_{i}\right)\right] \subset \operatorname{St}_{K}\left(y_{i}\right) ; \\
& F\left[\bar{U}\left(y_{i}, \eta_{i}\right)\right] \cap \bar{U}\left(y_{i}, \eta_{i}\right)=\phi, i=1,2, \cdots .
\end{aligned}
$$

We choose a path $P_{i}=\left[F\left(y_{i}\right), A_{i}, y_{i}, B_{i}, g\left(y_{i}\right)\right]$ in $\operatorname{St}_{K}\left(y_{i}\right)$, parametrized by length, such that points $A$ and $B$ belong to the maximal simplices of $K$. Defining the map $F_{1}:|K| \rightarrow|K|$ as:

$$
F_{1}(x)=\left[\begin{array}{l}
F(x), x \in|K|-\bigcup_{i} U\left(y_{i}, \eta_{i}\right) ; \\
F\left[\left(\frac{2 \rho\left(x, y_{i}\right)}{\eta_{i}}-1\right) x+\left(2-\frac{2 \rho\left(x, y_{i}\right)}{\eta_{i}}\right) y_{i}\right] \\
\eta_{i} / 2 \leqq \rho\left(x, y_{i}\right) \leqq \eta_{i} ; \\
P_{i}\left(1-\frac{2 \rho\left(x, y_{i}\right)}{\eta_{i}}\right), 0 \leqq \rho\left(x, y_{i}\right) \leqq \eta_{i} / 2,
\end{array}\right.
$$


$F_{1}$ satisfies the conditions of this lemma.

THeOREM 1. Assume that $K$ is an infinite and locally finite complex and that $L$ is a two-dimensionally connected infinite subcomplex which has the boundary $\dot{L}$ consisting of some vertices of $K$. Assume that $f:|K| \rightarrow|K|$ is a map and that each fixed point of $f$ on $|L|-|\dot{L}|$ is isolated and lies in a maximal simplex of $L$. Then there exists a map $F:|K| \rightarrow|K|$ which has the following two properties:

(a) $F \cong f \operatorname{rel}|\overline{K-L}|$; and,

(b) $F$ has no fixed points on $|L|-|\dot{L}|$. If $f$ satisfies $S(K)$ on $|K|$ then $F$ also satisfies $S(K)$ on $|K|$.

Proof. The basic method of constructing $F$ from $f$ is to push a fixed point of $f$ further away on $L$. First we choose the route of pushing the fixed point of $f$. We construct a one-dimensional complex $R$ such that there exists a one-to-one correspondence $g$ from all the maximal simplices of $L$ to all the vertices of $R$, where two vertices $g\left(\sigma_{1}\right)$ and $g\left(\sigma_{2}\right)$ constitute a one-dimensional simplex in $R$ if, and only if, $\sigma_{1}$ and $\sigma_{2}$ have a common face of dimension greater than zero. Then $R$ is a connected, infinite and locally finite complex. We choose a tree $S$ in $R$ which is a simply connected subcomplex of $R$ and contains all the vertices of $R$.

We now construct a function $N$ on the simplices of $S$ by inductive definition. In complex $S$, if a vertex $\tau^{0}$ is a face of a single one-dimensional simplex $\tau^{1}$ only, then we define $N\left(\tau^{0}\right)=1$ and $N\left(\tau^{1}\right)=1$. Evidently, $S-N^{-1}(1)$ is a subcomplex of $S$. In complex $S-\bigcup_{r=1}^{i-1} N^{-1}(r)$, if a vertex $\tau^{0}$ is a face of a single one-dimensional simplex $\tau^{1}$ only, then we define $N\left(\tau^{0}\right)=i$ and $N\left(\tau^{1}\right)=i$. Evidently, $S-\bigcup_{r=1}^{i} N^{-1}(r)$ is a subcomplex of $S$. Let $T=S-\bigcup_{r=1}^{\infty} N^{-1}(r)$. If $T$ is nonempty, then $T$ is a subcomplex of $S$ and we define $N(\tau)=$ 0 for all $\tau \in T$. As a result, function $N$ has the following properties (1) and (2):

(1) $S-\bigcup_{r=1}^{i} N^{-1}(r)$ is simply connected, for $i=1,2, \cdots$.

(2) if $\tau^{0}$ is a vertex of $S-T$, then there exists another vertex $\sigma^{0}$ of $S$ such that we have either $N\left(\sigma^{0}\right)>N\left(\tau^{0}\right)$ or $N\left(\sigma^{0}\right)=0$, where $\tau^{0}$ and $\sigma^{0}$ constitute a one-dimensional simplex of $S$.

(3) If $T$ is nonempty, from (1) we know that $T$ is a simply connected and infinite subcomplex of $S$. (See Fig. 1). In this case, we pick a vertex $A$ in $T$ and construct a function $V$ on all the vertices of $T$ as follows: For a vertex $\tau^{0}, V\left(\tau^{0}\right)$ is defined to be the least number of edges from $A$ to $\tau^{0}$ in $T$. In this case the property (4) is similar to property (2):

(4) if $\tau^{0}$ is a vertex of $T$, then there exists another vertex 


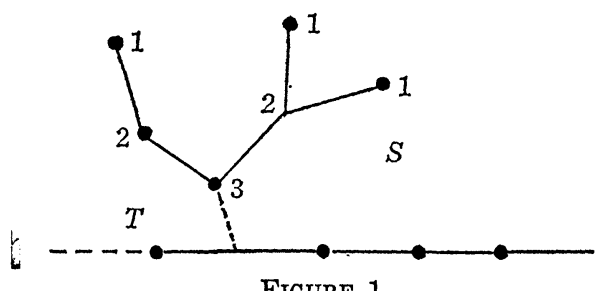

$\sigma^{0}$ of $T$ such that $V\left(\sigma^{0}\right)>V\left(\tau^{0}\right)$, where $\tau^{0}$ and $\sigma^{0}$ constitute a onedimensional simplex of $T$.

Based on the Lemma 1 and property (2), we can move the fixed points of $f$ from $g^{-1} N^{-1}(1)$ to $\left\{g^{-1} N^{-1}(r) / r=0\right.$ or $\left.r>1\right\}$, and subsequently move the fixed points of $f$ from $g^{-1} N^{-1}(i)$ to $\left\{g^{-1} N^{-1}(r) / r=\right.$ 0 or $r>i$, and so on, thereby moving all the fixed points of $f$ to $\left\{g^{-1} N^{-1}(0)\right\}$. Further, based on the Lemma 1 and property (4), we can move the fixed points of $f$ from $g^{-1} V^{-1}(1)$ to $\left\{g^{-1} V^{-1}(r) / r>1\right\}$, and subsequently move the fixed points of $f$ from $g^{-1} V^{-1}(i)$ to $\left\{g^{-1} V^{-1}(\boldsymbol{r}) / \boldsymbol{r}>i\right\}$ and so on. Finally, we get a map $F$ such that $F \cong f \operatorname{rel} \overline{|K-L|}$ and $F$ has no fixed points on $|L|-|\dot{L}|$.

From the Theorem 1 we deduce:

THEOREM 2. Suppose $K$ is an infinite and locally finite twodimensionally connected complex, then the least number of the fixed points of any mapping class from $|K|$ to itself is zero.

Definition 1 . Let $K$ be a locally finite complex and $M_{i}, i=1$, $2, \cdots$, be all its maximal two-dimensionally connected finite subcomplexes, thus the boundary $\dot{M}_{i}$ consists of some vertices of $K$. Denote $\dot{M}(K)=\mathrm{U}_{i} \dot{M}_{i}, \dot{M}(K)$ is called the welding set of $K$. A good displacement is a map $g: \dot{M}(K) \rightarrow|K|$ such that:

(1) $g(a) \in\left|\mathrm{St}_{K}(a)\right|$, for all $a \in \dot{M}(K)$; and,

(2) if $g$ has no fixed points in $\dot{M}_{i}$, then the number of points in $\dot{M}_{i}$ whose images under $g$ are outside $\left|M_{i}\right|$ is exactly $\chi\left(M_{i}\right)$.

THEOREM 3. Let $K$ be a locally finite complex, then the least number $m(K)$ of fixed points of the identity mapping class is equal to the least number of fixed points $n(K)$ of all the good displace-

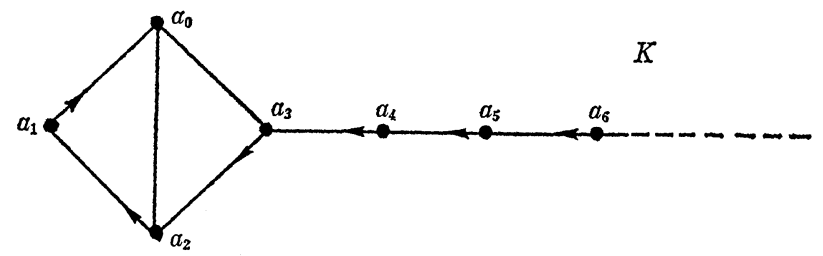

Figure 2 
ments.

In Lemma 4 we shall prove $m(K) \leqq n(K)$ and in Lemma 5 we shall prove $m(K) \geqq n(K)$.

EXAMPLE 1. In Fig. 2, the welding set $\dot{M}(K)$ of $K$ is $\left\{a_{0}, a_{1}\right.$, $\left.a_{2}, \cdots\right\}$ and the arrows represent a good displacement which has the least fixed points. From Theorem 3 we have $m(K)=1$. Replacing each 1-dimensional closed simplex $\tau_{i}=a_{j} a_{k}$ of $K$ by a 2-dimensionally connected complex $M_{i}$, such that $\dot{M}_{i}=\left\{a_{j}, a_{k}\right\}$, we get a complex $K_{1}$ with $\dot{M}\left(K_{1}\right)=\dot{M}(K)$. If each $M_{i}$ is an $n$-dimensional closed simplex, then $m\left(K_{1}\right)=1$ results from Theorem 3 ; if for each $M_{i}$, either $\chi\left(M_{i}\right)>2$ or $\chi\left(M_{i}\right)<0$, then $m\left(K_{1}\right)=\infty$ from Theorem 3.

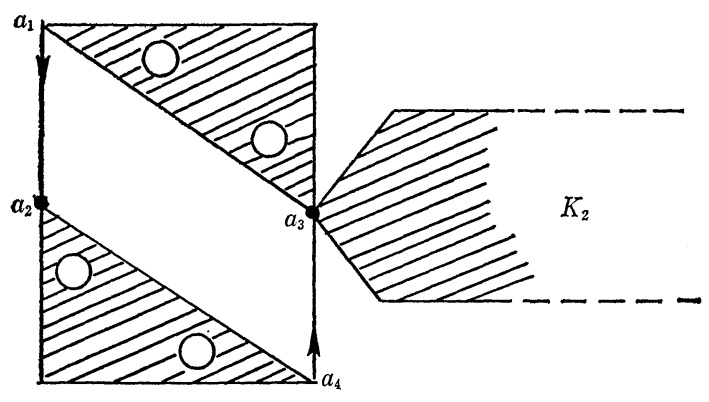

FiguRe 3

ExAmple 2. In Fig. 3, the welding set $\dot{M}\left(K_{2}\right)_{\dot{2}}^{\text {of }} K_{2}$ is $\left\{a_{1}, a_{2}\right.$, $\left.a_{3}, a_{4}\right\}$, and the arrows represent a good displacement which has least fixed points. From Theorem 3 we have $m\left(K_{2}\right)=2$.

Lemma 4. If $g$ is a good displacement of $K$, there will be a $\operatorname{map} G:|K| \rightarrow|K|$ such that:

(1) $G(x)=g(x)$, for all $x \in \dot{M}(K)$;

(2) $G$ satisfies $S(K)$ on $|K|$; and,

(3) $G$ has no fixed points on $|K|-\dot{M}(K)$.

Proof. Applying Lemma 3, we get a map $F_{1}:|K| \rightarrow|K|$ that has the following three properties:

(1) $F_{1}$ satisfies $S(K)$ on $|K|$;

(2) $F_{1}(x)=g(x)$, for all $x \in \dot{M}(K)$; and,

(3) each fixed point of $F_{1}$ on $|K|-\dot{M}(K)$ is isolated and lies in a maximal simplex of $K$.

From Theorem 1, there exists a map $F$, such that, $F$ satisfies $S(K)$ on $|K|, F \cong F_{1}:|K| \rightarrow|K|$ rel $\bigcup_{i} M_{i}$, and $F$ in $\left|K-\bigcup_{i} M_{i}\right|$ has no fixed points. 
Since $g$ is a good displacement, if $g$ has no fixed points on $\dot{M}_{i}$, the fixed point index of $F$ in $M_{i}$ is zero, (see Appendix). From Lemma 1 , we may move all the fixed points of $F$ on $\left|M_{i}-\dot{M}_{i}\right|$ to any single point and then cancel this fixed point (see page 123 of [2]). If the map $g$ in $\dot{M}_{i}$ has a fixed point $A$, then applying Lemma 1 as many times as necessary we may move all the fixed points of $F$ on $\left|M_{i}\right|-\left|\dot{M}_{i}\right|$ to $A$ and finally get the map $G$.

In order to prove $n(K) \leqq m(K)$, we introduce the concept of fixed point classes on an open subset.

DEFINITION 2. Assume that $U$ is an open subset of the polyhedron $|K|$ of a locally finite complex $K$ where $\bar{U}$ is compact. Assume that a map $f: \bar{U} \rightarrow|K|$ has no fixed point on $\dot{U}$. Fixed points $a$ and $b$ of $f$ in $U$ are said to belong to the same fixed point class if there is a path $P(t)$ on $U$ such that $P(0)=a, P(1)=b$, and $f[P(t)] \cong P(t)$ rel $\{a, b\}$ on $|K|$.

We may define the index of fixed point classes. The fixed point class with a nonzero index is called an essential fixed point class. The number of essential fixed point classes of $f$ on $U$ is finite.

Definition 3. Suppose that a homotopy $f_{t}: \bar{U} \rightarrow|K|, 0 \leqq t \leqq 1$, has no fixed points on $\dot{U}, f_{0}(a)=a, f_{1}(b)=b$ and that $P(t)$ is a path on $U$ connecting $a$ and $b$ such that

$$
f_{t}[P(t)] \cong P(t) \text { rel }\{a, b\} \text { on }|K| \text {. }
$$

Thus we say there is a homotopy correspondence between the fixed point class of $f_{0}$ on $U$ which contains $a$ and the fixed point class of $f_{1}$ on $U$ which contains $b$. This homotopy correspondence is a one-to-one correspondence between all the essential fixed point classes of $f_{0}$ and all the essential fixed point classes of $f_{1}$. The corresponding classes have the same index.

Lemma 5. Suppose that $K$ is a locally finite complex and that $1 \cong f:|K| \rightarrow|K|$. Then there exists a good displacement $g$ such that the number of fixed points of $g$ is not greater than the number of fixed points of $f$.

\section{Proof.}

(1) If $f$ has fixed points on $\left|M_{s}\right|-\dot{M}_{s}$ for some $M_{s}$ of $K$, we arbitrarily assign a point in $\dot{M}_{s}$. The set of the assigned points and the fixed points of $f$ on $\dot{M}(K)$ are denoted by $\left\{b_{1}, b_{2}, \cdots\right\}$, then the number of points in $\left\{b_{1}, b_{2}, \cdots\right\}$ is not greater than the number 
of fixed points of $f$. We write

$$
\left\{c_{1}, c_{2}, \cdots\right\}=\dot{M}(K)-\left\{b_{1}, b_{2}, \cdots\right\} .
$$

Let $f_{t}: 1 \cong f:|K| \rightarrow|K|$, then $f_{t}\left(c_{i}\right)$ is a path from $c_{i}$ to $f\left(c_{i}\right)$. Based on $f_{t}\left(c_{i}\right)$, we can construct a path $Q_{i}(t)=\alpha_{1}^{i} \cdot \alpha_{2}^{i} \cdots \alpha_{h}^{i} \cdot \beta^{i}$ that has the following four properties:

(a) for $j=1,2, \cdots, h$, there are points $b_{j}^{i}, c_{j}^{i} \in \mathrm{St}_{K}\left(c_{i}\right)$ and polygonal arcs $\theta_{j}^{i}$ from $b_{j}^{i}$ to $c_{j}^{i}$ not containing $c_{i}$ (see Fig. 4) such that

$$
\alpha_{j}^{i}=\left[c_{i}, b_{j}^{i}\right] \cdot \theta_{j}^{i} \cdot\left[c_{j}^{i}, c_{i}\right], j=1,2, \cdots, h ;
$$

(b) $\beta^{i}=\left[c_{i}, b_{h+1}^{i}\right] \cdot \theta_{h+1}^{i}$;

where $b_{h+1}^{i} \in \operatorname{St}_{K}\left(c_{i}\right)$ and $\theta_{h+1}^{i}$ is a polygonal are from $b_{h+1}^{i}$ to $f\left(c_{i}\right)$ not containing $c_{i}$;

(c) $\alpha_{1}^{i} \cdot \alpha_{2}^{i} \cdots \alpha_{r}^{i} \not 1, r=1, \cdots, h$;

(d) $f_{t}\left(c_{i}\right) \cong Q_{i}(t) \operatorname{rel}\left\{c_{i}, f\left(c_{i}\right)\right\}, 1=1,2, \cdots$.

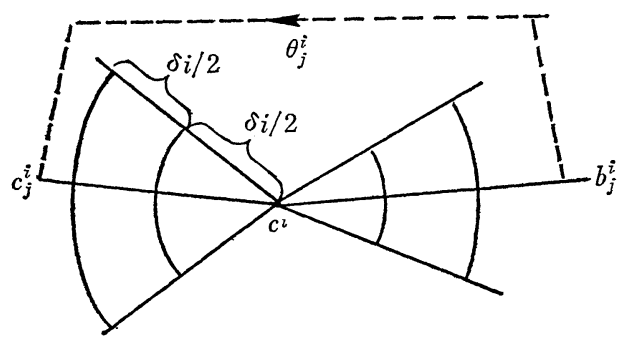

FIGURE 4

From the homotopy extension theorem, there is another homotopy $f_{i}: 1 \cong f:|K| \rightarrow|K|$ with $f_{t}\left(c_{i}\right)=Q_{i}(t), i=1,2, \cdots$.

(2) For each $c_{i}$, we choose a sufficiently small $\delta_{i}>0$ such that:

(a) $\bar{U}\left(c_{i}, \delta_{i}\right) \subset \operatorname{St}_{K}\left(c_{i}\right)$;

(b) $\bar{U}\left(c_{i}, \delta_{i}\right) \cap \bar{U}\left(c_{j}, \delta_{j}\right)=\phi, i \neq j$;

(c) $\bar{U}\left(c_{i}, \delta_{i}\right) \cap \theta_{j}^{j}=\phi, j=1, \cdots, h+1$;

(d) $b_{j}^{i} \in|K|-\mathrm{U}_{i} U\left(c_{i}, \delta_{i}\right), j=1, \cdots, h+1$,

$$
c_{j}^{i} \in|K|-\mathrm{U}_{i} U\left(c_{i}, \delta_{i}\right), j=1, \cdots, h .
$$

We define a map $F:|K| \rightarrow|K|$ by

$$
F(x)=\left[\begin{array}{l}
x, \quad x \in|K|-U U\left(c_{i}, \delta_{i}\right), \\
{\left[\frac{2 \rho\left(x, c_{i}\right)}{\delta_{i}}-1\right] x+\left[2-\frac{2 \rho\left(x, c_{i}\right)}{\delta_{i}}\right] c_{i},} \\
\delta_{i} / 2 \leqq \rho\left(x, c_{i}\right) \leqq \delta_{i}, \\
Q_{i}\left[1-\frac{2 \rho\left(x, c_{i}\right)}{\delta_{i}}\right], 0 \leqq \rho\left(x, c_{i}\right) \leqq \delta_{i} / 2,
\end{array}\right.
$$

thus 


$$
F \cong f \operatorname{rel}\left\{c_{1}, c_{2}, \cdots\right\} \text {. }
$$

(3) The fixed point set of $F$ on $|K|$ is $N_{1} \cup N_{2}$, where

(a) $N_{1}=|K|-\mathrm{U}_{i} U\left(c_{i}, \delta_{i}\right)$;

(b) $N_{2}=\bigcup_{i}\left\{d_{1}^{i}, d_{2}^{i}, \cdots, d_{h+1}^{i}, e_{1}^{i}, e_{2}^{i}, \cdots, e_{h}^{i}\right\}$, where $d_{j}^{i} \in\left(c_{i}, b_{j}^{i}\right), j=$ $1,2, \cdots, h+1$, and $e_{j}^{i} \in\left(c_{j}^{i}, c_{i}\right), j=1, \cdots, h$; moreover,

(c) $\delta_{i} / 2>\rho\left(c_{i}, d_{1}^{i}\right)>\rho\left(c_{i}, e_{1}^{i}\right)>\rho\left(c_{i}, d_{2}^{i}\right)>\rho\left(c_{i}, e_{2}^{i}\right) \cdots \rho\left(c_{i}, d_{h+1}^{i}\right)>0$.

(4) If $\dot{M}_{s} \subset\left\{c_{1}, c_{2}, \cdots\right\}$, then $F$ on $\dot{M}_{s}$ has no fixed points, and we can discuss the fixed point classes of $F$ on $\left|M_{s}\right|-\dot{M}_{s}$.

(a) If $d_{1}^{i} \in\left|M_{s}\right|$, then $d_{1}^{i}$ and $N_{1} \cap\left|M_{s}\right|$ belong to the same fixed point class, the reason being $b_{1}^{i} \in N_{1} \cap\left|M_{s}\right|$, and $F\left(\left[b_{1}^{i}, d_{1}^{i}\right]\right) \cdot\left[d_{1}^{i}, b_{1}^{i}\right]=$ $\left[b_{1}^{i}, c_{i}\right] \cdot\left[c_{i}, d_{1}^{i}\right] \cdot\left[d_{1}^{i} \cdot b_{1}^{i}\right] \cong 1$. Excluding these $\left(\bigcup_{i} d_{1}^{i}\right) \cap\left|M_{s}\right|$, each fixed point of $N_{2} \cap\left|M_{s}\right|$ does not belong to the same fixed point class as $N_{1} \cap\left|M_{s}\right|$. This fact will be proved in (b) and (c).

(b) Suppose $b(t)$ is a path from $b_{r}^{i}$ to $d_{r}^{i}(r>1)$ in $\left|M_{s}\right|-\dot{M}_{s}$, then there exists a loop $\beta$ based at $b_{r}^{i}$ such that $\beta \subset\left|M_{s}\right| \cap N_{1}$ and $b(t) \cong \beta \cdot\left[b_{r}^{i}, d_{r}^{i}\right] \operatorname{rel}\left\{b_{r}^{i}, d_{r}^{i}\right\}$. Hence,

$$
\begin{aligned}
F(b(t)) & \cdot b(t)^{-1} \cong F\left(\beta \cdot\left[b_{r}^{i}, d_{r}^{i}\right]\right) \cdot\left[d_{r}^{i}, b_{r}^{i}\right] \cdot \beta^{-1} \\
& =\beta \cdot F\left(\left[b_{r}^{i}, d_{r}^{i}\right]\right) \cdot\left[d_{r}^{i}, b_{r}^{i}\right] \cdot \beta^{-1} \\
& \cong \beta \cdot\left[b_{r}^{i}, c_{i}\right] \cdot \alpha_{1}^{i} \cdot \alpha_{2}^{i} \cdots \alpha_{r-1}^{i} \cdot\left[c_{i}, d_{r}^{i}\right] \cdot\left[d_{r}^{i}, b_{r}^{i}\right] \cdot \beta^{-1} \\
& \cong \beta \cdot\left[b_{r}^{i}, c_{i}\right] \cdot \alpha_{1}^{i} \cdot \alpha_{2}^{i} \cdots \alpha_{r-1}^{i} \cdot\left[c_{i}, b_{r}^{i}\right] \cdot \beta^{-1} \not 1
\end{aligned}
$$

on $|K|$; because we required that $\alpha_{1}^{i} \cdot \alpha_{2}^{i} \cdots \alpha_{r-1}^{i} \not 1$.

(c) Similarly, suppose $b(t)$ is a path from $c_{r}^{i}$ to $e_{r}^{i}(r \geqq 1)$ in $\left|M_{s}\right|-\dot{M}_{s}$, then there exists a loop $\beta$ of $c_{r}^{i}$ such that $\beta \subset\left|M_{s}\right| \cap N_{1}$ and $b(t) \cong \beta \cdot\left[c_{r}^{i}, e_{r}^{i}\right] \operatorname{rel}\left\{c_{r}^{i}, d_{r}^{i}\right\}$. Hence,

$$
b(t) \not F(b(t)) \text { on }|K| \text {. }
$$

(d) Since $f$ in $\left|M_{s}\right|$ has no fixed points, the index of each fixed point class of $F$ on $\left|M_{s}\right|-\dot{M}_{s}$ is zero, in particular the index of the fixed point class containing $\left|M_{s}\right| \cap N_{1}$ is zero.

(5) We define a map $g: \dot{M}(K) \rightarrow|K|$ as follows:

$$
g\left(c_{i}\right)=b_{1}^{i}, i=1,2, \cdots ;
$$

and,

$$
g\left(b_{j}\right)=b_{j}, j=1,2, \cdots .
$$

Consider the fixed point class of $F$ on $\left|M_{s}\right|-\dot{M}_{s}$. Since the index of the fixed point class containing $\left|M_{s}\right| \cap N_{1}$ is zero, then there are exactly $\chi\left(M_{s}\right)$ points in $\dot{M}_{s}$, whose images under $g$ are outside $\left|M_{s}\right|$ (see Appendix), so $g$ is a good displacement.

APPENDIX. The proof of Lemma 2 of [7] (it was published previously in Chinese). 
Lemma. Assume that $K$ is a locally finite complex and $M$ is a maximal two-dimensionally connected finite subcomplex. Assume that $g: \dot{M} \rightarrow|K|$ is a map such that

(1) $g(a) \in\left|\mathrm{St}_{K}(a)\right|$, for all $a \in \dot{M}$;

(2) $g(a) \neq a$, for all $a \in \dot{M}$, and $g$ maps $\chi_{g}$ points of $\dot{M}$ outside of $|M|$; and

(3) $[a, g(a)] \cap[b, g(b)]=\varnothing$, for any $a, b \in \dot{M}$.

If a map $F:|M| \rightarrow|K|$ has the following two properties:

(i) $F(a)=g(a)$, for all $a \in \dot{M}$; and

(ii) $F$ satisfies $S(K)$ on $|M|$, then $J(F,|M|-\dot{M})$ the index of fixed points of $F$ on $|M|-\dot{M}$, equals $\chi(M)-\chi_{g}$.

Proof. We denote the points of $\dot{M}$ by $a_{j}, j=1, \cdots, r$. Assume that $g\left(a_{j}\right) \notin|M|$ for $j=1,2, \cdots, \chi_{g}$ and $g\left(a_{j}\right) \in|M|$ for $j=\chi_{g}+1$, $\chi_{g}+2, \cdots, r$. First choose $b_{j}, j=1, \cdots, \chi_{g}$, so that $g\left(a_{j}\right) \in\left(a_{j}, b_{j}\right)$, $\left[a_{j}, b_{j}\left[\subset \mathrm{St}_{K}\left(a_{j}\right) \subset|K|\right.\right.$ and that any two segments of $\left\{\left[a_{j}, b_{j}\right] \mid j=\right.$ $\left.1, \cdots, \chi_{g}\right\}$ are disjoint (from property 3). Let $K^{\prime}$ denote the complex composed of $M$ and $\left[a_{j}, b_{j}\right], j=1, \cdots, \chi_{g}$. Let $g^{\prime}$ be the map $g$ considered as a map from $\dot{M}$ to $\left|K^{\prime}\right|$. Applying Lemma 3 to $g^{\prime}$ and $K^{\prime}$, we know there exists a map $G_{1}:\left|K^{\prime}\right| \rightarrow\left|K^{\prime}\right|$ such that $G_{1}\left(a_{j}\right)=$ $g^{\prime}\left(a_{j}\right)=g\left(a_{j}\right), j=1, \cdots, r, G_{1}$ satisfies $S\left(K^{\prime}\right)$ on $\left|K^{\prime}\right|$. Define a map $G_{2}:\left|K^{\prime}\right| \rightarrow\left|K^{\prime}\right|$ as follows:

$$
G_{2}(x)=\left\{\begin{array}{l}
G_{1}(x), x \in|M| ; \\
g\left(a_{j}\right), x \in\left[a_{j}, b_{j}\right], j=1,2, \cdots, \chi_{g} .
\end{array}\right.
$$

Since $G_{2}$ is homotopic to the identity map,

$$
J\left(G_{2}, K^{\prime}\right)=\chi\left(K^{\prime}\right)=\chi(M)
$$

by "Axiom 4" on page 52 of [2]. Since $G_{2}$ on $\left[a_{j}, b_{j}\right], j=1, \cdots, \chi_{g}$, only has one fixed point $g\left(a_{j}\right)$ of index +1 , we obtain

$$
J\left(G_{2},|M|-\dot{M}\right)+\chi_{g}=\chi(M)
$$

i.e.,

$$
J\left(G_{2},|M|-\dot{M}\right)=\chi(M)-\chi_{g} .
$$

Now, denote the inclusion map of $\left|K^{\prime}\right|$ into $|K|$ by $I$, and let $G_{3}=$ $I G_{2}:|M| \rightarrow|K|$. We have $J\left(G_{3},|M|-\dot{M}\right)=\chi(M)-\chi_{g}$. Finally, recall the map $F:|M| \rightarrow|K|$ assumed in this lemma. Since it has the two properties listed, the map $\alpha(x, F(x), t)$ ([2], pages 124-126), for $x \in|M|, 0 \leqq t \leqq 1$, is a homotopy equivalence between the identify mapping $I$ and $F$. Since $G_{1}$ satisfies $S\left(K^{\prime}\right)$ on $\left|K^{\prime}\right|$, it also satisfies $S(K)$ on $\left|K^{\prime}\right|$. Moreover, $G_{3}$ satisfies $S(K)$ on $|M|$. So $\alpha\left(x, G_{3}(x), t\right)$, 
$x \in|M|, 0 \leqq t \leqq 1$, is a homotopy equivalence from $I$ to $G_{3}$. Furthermore $\alpha(x, F(x), t)=\alpha\left(x, G_{3}(x), t\right)$ when $x \in \dot{M}, 0 \leqq t \leqq 1$. Consequently, employing the homotopy extension theorem on $|M|, F \cong$ $G_{3}$ rel $\dot{M}$. Thus we get the conclusion of this lemma: $J(F,|M|-\dot{M})=$ $J\left(G_{3} ;|M|-\dot{M}\right)=\chi(M)-\chi_{g}$.

\section{REFERENCES}

1. P. Alexandroff and H. Hopf, Topologie, Springer, Berlin, 1935.

2. R. F. Brown, the Lefschetz Fixed Point Theorem, Scott, Foresman and Co., 1971.

3. E. Fadell, Recent results in the fixed point theory of continuous maps, Bull. Amer. Math. Soc., 76 (1970), 10-29.

4. B. J. Jiang, Estimation of the Nielsen numbers, Acta Math. Sinica, 14 (1964), 304-312. (=Chinese Math., 5 (1964), 330-339.)

5. T. H. Kiang, The Theory of Fixed Point Classes, Scientific Press, Peking, 1979.

6. G. H. Shi, On the least numbers of fixed points and Nielsen numbers, Acta Math. Sinica, 16 (1966), 223-232. (=Chinese Math., 8 (1966), 234-243).

7. - The least number of fixed points of the identity mapping class, Acta Math. Sinica, 18 (1975), 192-202.

Received August 7, 1980 and in revised form February 4, 1981.

\#8 Shiang-Chun Road

P. O. BoX 366 BEIJING

People's Republic of China 



\title{
PACIFIC JOURNAL OF MATHEMATICS
}

\section{EDITORS}

\author{
DONALD BABBITT (Managing Editor) \\ University of California \\ Los Angeles, CA 90024 \\ Hugo Rossi \\ University of Utah \\ Salt Lake City, UT 84112 \\ C. C. MOORE and ARthur Agus \\ University of California \\ Berkeley, CA 94720
}

J. DUGUNDJI

Department of Mathematics

University of Southern California

Los Angeles, CA 90007

R. FINN and J. MILGRAM

Stanford University

Stanford, CA 94305

\section{ASSOCIATE EDITORS}
R. ARENS
E. F. BeCKENBACH
B. H. NeumanN
F. WoLF
K. YoSHIDA

\section{SUPPORTING INSTITUTIONS}

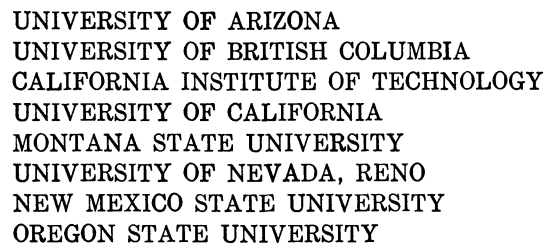

UNIVERSITY OF ARIZONA

UNIVERSITY OF BRITISH COLUMBIA

CALIFORNIA INSTITUTE OF TECHNOLOGY

UNIVERSITY OF CALIFORNIA

MONTANA STATE UNIVERSITY

UNIVERSITY OF NEVADA, RENO

NEW MEXICO STATE UNIVERSITY

OREGON STATE UNIVERSITY

\author{
UNIVERSITY OF OREGON \\ UNIVERSITY OF SOUTHERN CALIFORNIA \\ STANFORD UNIVERSITY \\ UNIVERSITY OF AAWAII \\ UNIVERSITY OF TOKYO \\ UNIVERSITY OF UTAH \\ WASHINGTON STATE UNIVERSITY \\ UNIVERSITY OF WASHINGTON
}

The Supporting Institutions listed above contribute to the cost of publication of this Journal, but they are not owners or publishers and have no responsibility for its content or policies,

Mathematical parers intended for publication in the Pacific Journal of Mathematics should be in typed form or offset-reproduced, (not dittoed), double spaced with large margins. Please do not use built up fractions in the text of the manuscript. However, you may use them in the displayed equations. Underline Greek letters in red, German in green, and script in blue. The first paragraph or two must be capable of being used separately as a synopsis of the entire paper. Please propose a heading for the odd unmbered pages of less than 35 characters. Manuscripts, in triplicate, may be sent to any one of the editors. Please classify according to the scheme of Math. Reviews, Index to Vol. 39. Supply name and address of author to whom proofs should be sent. All other communications should be addressed to the managing editor, or Elaine Barth, University of California, Los Angeles, California, 90024.

50 reprints to each author are provided free for each article, only if page charges have been substantially paid. Additional copies may be obtained at cost in multiples of 50 .

The Pacific Journal of Mathematics is issued monthly as of January 1966, Regular subscription rate: $\$ 114.00$ a year (6 Vol., 12 issues). Special rate: $\$ 57.00$ a year to individual members of supporting institution.

Subscriptions, orders for numbers issued in the last three calendar years, and changes of address shoud be sent to Pacific Journal of Mathematics, P.O. Box 969, Carmel Valley, CA 93924, U.S.A. Old back numbers obtainable from Kraus Periodicals Co., Route 100, Millwood, NY 10546.

PUBLISHED BY PACIFIC JOURNAL OF MATHEMATICS, A NON-PROFIT CORPORATION

Printed at Kokusai Bunken Insatsusha (International Academic Printing Co., Ltd.). 8-8, 3-chome, Takadanobaba, Shinjuku-ku, Tokyo 160, Japan. 


\section{Pacific Journal of Mathematics}

\section{Vol. 103, No. $2 \quad$ April, 1982}

Alberto Alesina and Leonede De Michele, A dichotomy for a class of positive

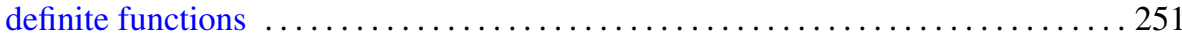

Kahtan Alzubaidy, Rank 2 -groups, $p>3$, and Chern classes . . . . . . . . . . 259

James Arney and Edward A. Bender, Random mappings with constraints on

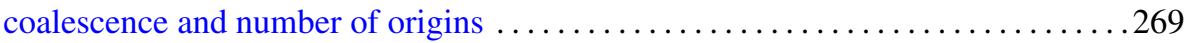

Bruce C. Berndt, An arithmetic Poisson formula . . . . . . . . . . . . . . . 295

Julius Rubin Blum and J. I. Reich, Pointwise ergodic theorems in 1.c.a. groups . . . 301

Jonathan Borwein, A note on $\varepsilon$-subgradients and maximal monotonicity . . . . . . . 307

Andrew Michael Brunner, Edward James Mayland, Jr. and Jonathan Simon,

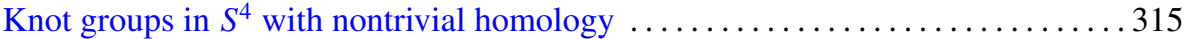

Luis A. Caffarelli, Avner Friedman and Alessandro Torelli, The two-obstacle

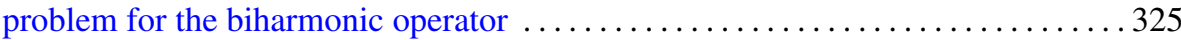

Aleksander Całka, On local isometries of finitely compact metric spaces . . . . . . 337

William S. Cohn, Carleson measures for functions orthogonal to invariant

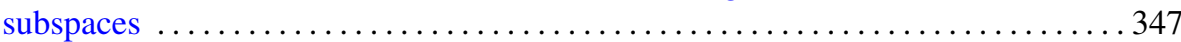

Roger Fenn and Denis Karmen Sjerve, Duality and cohomology for one-relator

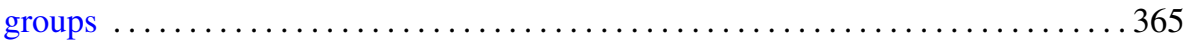

Gen Hua Shi, On the least number of fixed points for infinite complexes . . . . . . . 377

George Golightly, Shadow and inverse-shadow inner products for a class of linear

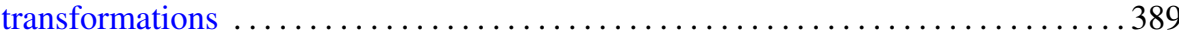

Joachim Georg Hartung, An extension of Sion's minimax theorem with an

application to a method for constrained games $\ldots \ldots \ldots \ldots \ldots \ldots \ldots \ldots \ldots \ldots . \ldots 4$

Vikram Jha and Michael Joseph Kallaher, On the Lorimer-Rahilly and

Johnson-Walker translation planes

Kenneth Richard Johnson, Unitary analogs of generalized Ramanujan sums .

Peter Dexter Johnson, Jr. and R. N. Mohapatra, Best possible results in a class of inequalities

Dieter Jungnickel and Sharad S. Sane, On extensions of nets

Johan Henricus Bernardus Kemperman and Morris Skibinsky, On the

characterization of an interesting property of the arcsin distribution ...

Karl Andrew Kosler, On hereditary rings and Noetherian $V$-rings

William A. Lampe, Congruence lattices of algebras of fixed similarity type. II . . . . 475

M. N. Mishra, N. N. Nayak and Swadeenananda Pattanayak, Strong result for real zeros of random polynomials

Sidney Allen Morris and Peter Robert Nickolas, Locally invariant topologies on free groups

Richard Cole Penney, A Fourier transform theorem on nilmanifolds and nil-theta functions

Andrei Shkalikov, Estimates of meromorphic functions and summability theorems

László Székelyhidi, Note on exponential polynomials

William Thomas Watkins, Homeomorphic classification of certain inverse limit spaces with open bonding maps $\ldots \ldots \ldots \ldots \ldots \ldots \ldots \ldots$

David G. Wright, Countable decompositions of $E^{n}$

Takayuki Kawada, Correction to: "Sample functions of Pólya processes" .

Z. A. Chanturia, Errata: "On the absolute convergence of Fourier series of the 